\title{
The AGN fraction-velocity dispersion relation in clusters of galaxies
}

\author{
P. Popesso ${ }^{1}$ and A. Biviano ${ }^{2}$ \\ 1 European Southern Observatory, Karl Scharzschild Strasse 2, 85748 Garching, Germay \\ e-mail: ppopesso@eso.org \\ 2 INAF - Osservatorio Astronomico di Trieste, via G. B. Tiepolo 11, 34143 Trieste, Italy
}

Received 18 August 2006 / Accepted 16 October 2006

ABSTRACT

\begin{abstract}
Context. Some previous investigations have found that the fraction $\left(f_{\mathrm{AGN}}\right)$ of active galactic nuclei (AGNs) is lower in clusters than in the field. This can result from the suppression of galaxy-galaxy mergers in high-velocity dispersion $\left(\sigma_{v}\right)$ clusters, if the formation and/or fueling of AGNs is directly related to the merging process.

Aims. We investigate the existence of a relation between $f_{\mathrm{AGN}}$ and $\sigma_{v}$ in galaxy clusters in order to shed light on the formation and evolution processes of AGNs and cluster galaxies.

Methods. Using data from the Sloan Digital Sky Survey we determine $f_{\mathrm{AGN}}$ and $\sigma_{v}$ for the clusters in two samples, extracted from the catalogs of Popesso et al. (2006a, A\&A, in press) and Miller et al. (2005, AJ, 130, 968), and excluding clusters with significant evidence for substructures.

Results. We find a significant $f_{\mathrm{AGN}}-\sigma_{v}$ anti-correlation. Clusters with $\sigma_{v}$ lower and, respectively, higher than $500 \mathrm{~km} \mathrm{~s}^{-1}$ have AGN fractions of $0.21 \pm 0.01$ and $0.15 \pm 0.01$, on average. The $f_{\mathrm{AGN}}-\sigma_{v}$ relation can be described by a model that assumes $f_{\mathrm{AGN}}$ is proportional to the galaxies merging rate, plus a constant.

Conclusions. Since $f_{\mathrm{AGN}}$ increases with decreasing $\sigma_{v}$, AGNs are likely to have played a significant rôle in heating the intra-cluster medium and driving galaxy evolution in cluster precursors and groups.
\end{abstract}

Key words. galaxies: clusters: general - galaxies: active - galaxies: interactions - Galaxy: kinematics and dynamics

\section{Introduction}

Whether the fraction of active galactic nuclei (AGN hereafter) is environment-dependent or not is a matter of debate. Claims that clusters contain a lower fraction of AGNs ( $f_{\mathrm{AGN}}$ hereafter) than the field (Gisler 1978; Dressler et al. 1985, 1999; Hill \& Oegerle 1993; Rines et al. 2005) are in disagreement with the recent finding of Miller et al. (2003) that $f_{\mathrm{AGN}}$ does not vary with the local density. Such a discrepancy is explained by the finding of Kauffmann et al. (2004) that high-luminosity AGNs do avoid high-density regions, but low-luminosity AGNs do not; the latter dominate current AGN samples, but were normally missed in older AGN surveys. The quoted results were all based on optically-selected AGNs. X-ray observations have found a general over-density of X-ray emitting AGNs relative to field counts in the external regions of galaxy clusters (Henry \& Briel 1991; Lazzati et al. 1998; Cappi et al. 2001; Martini et al. 2006, and references therein). However, the observed over-densities do not necessarily imply that the fraction of cluster galaxies hosting an AGN is higher than the corresponding fraction of field galaxies.

A lower $f_{\mathrm{AGN}}$ in clusters relative to the field could be explained in terms of a decreased galaxy-galaxy merger efficiency in clusters. In fact, AGNs are thought to be fueled, or even formed, by the merger of gas-rich galaxies, which leads to rapid nuclear inflows of gas (e.g. Barnes \& Hernquist 1992; Springel et al. 2005, S05 hereafter). The decreased merger efficiency in clusters arises from the high relative velocities of cluster galaxies, since the merger rate scales roughly as $\sigma_{v}^{-3}$ for high values of $\sigma_{v}$, where $\sigma_{v}$ is the cluster velocity dispersion
(Mamon 1992; Makino \& Hut 1997). On the other hand, the low $\sigma_{v}$ and high density of groups make them a very favorable environment for galaxy-galaxy mergers, probably even more favorable than the field. An indication that this might indeed be the case comes from the high values of $f_{\mathrm{AGN}}$ (typically $\gtrsim 30 \%$ ) reported for galaxy compact groups (Coziol et al. 2000, 2004; Turner et al. 2001; Tovmassian et al. 2006), and from the relatively high $f_{\mathrm{AGN}}$ values found for "open clusters" in the early investigation of Gisler (1978).

In this letter the existence of a relation between cluster $f_{\mathrm{AGN}}$ and $\sigma_{v}$ is investigated. For this purpose, we use two samples of nearby (redshift $z<0.08$ ) galaxy clusters with data from the galaxy spectroscopic sample of the Sloan Digital Sky Survey (SDSS). In Sect. 2 we briefly describe our cluster samples, and how we determined $\sigma_{v}$ and $f_{\mathrm{AGN}}$. In Sect. 3 we analyze the relation between these two quantities. In Sect. 4 we discuss our results and provide our conclusions. Throughout this letter, we use $H_{0}=70 \mathrm{~km} \mathrm{~s}^{-1} \mathrm{Mpc}^{-1}$ in a flat cosmology with $\Omega_{0}=0.3$, and $\Omega_{\Lambda}=0.7$.

\section{The cluster sample, $\sigma_{v}$, and $f_{\mathrm{AGN}}$}

The optical data used in this paper are taken from the SDSS (see, e.g., York et al. 2000; Abazajian et al. 2004). We use two nearby cluster samples in our analysis, that of Popesso et al. (2006a, PBBR hereafter), and the C4 cluster catalog of Miller et al. (2005). Each cluster sample has its own merits. All clusters in the PBBR have X-ray counterparts (although not all of them are X-ray selected). The C4 catalog is entirely 
optically-selected, and has the advantage of having a well understood selection function.

PBBR have selected the cluster members by the method of Katgert et al. (2004), shown to be reliable by tests performed on clusters extracted from cosmological simulations (van Haarlem et al. 1997; Biviano et al. 2006). PBBR have determined virial masses, virial radii $\left(r_{200} \mathrm{~s}\right)$, and velocity dispersions $\left(\sigma_{v} \mathrm{~s}\right)$ using the cluster members with cluster-centric distances $\leq r_{200}$. We refer the reader to PBBR for all the relevant details. The sample of PBBR contains 137 clusters with at least 10 member galaxies within $r_{200}$. Ten member galaxies are considered to be the minimum number for a reliable determination of a cluster $\sigma_{v}$ (Girardi et al. 1993; Zabludoff \& Mulchaey 1998). Using the same methods of PBBR we select the cluster members in the $\mathrm{C} 4$ clusters, and then determine the $\sigma_{v}$ s of those clusters with at least 10 member galaxies within their $r_{200} \mathrm{~s}$ (609 clusters in total).

From both the PBBR and the $\mathrm{C} 4$ samples, we exclude those clusters with significant evidence for subclustering (probability $<1 \%$ ), according to the test of Dressler \& Shectman (1988). In this way we reduce the occurrence of very wrong $\sigma_{v}$-estimates (see, e.g., van Haarlem et al. 1997; Biviano et al. 2006). There are 17 and, respectively, 115 clusters with subclustering in the PBBR and C4 samples.

We use all galaxies from the SDSS spectroscopic sample, with no magnitude limit, to determine the cluster velocity dispersion, since there is no evidence for segregation of cluster galaxies in velocity space, apart for the very bright galaxies (Biviano et al. 1992). However, the fraction of AGN galaxies does depend on the galaxy mass (Kauffmann et al. 2003), therefore we need to sample the cluster galaxy populations down to the same absolute magnitude limit, that we set to $M_{r}=-20.0$. We then select only those clusters that are sampled deeper than this limit, i.e. those for which the limiting magnitude $r=17.77$ of the SDSS spectroscopic survey corresponds to an absolute magnitude $M_{r} \geq$ -20.0 , and with $>5$ members with $M_{r} \leq-20.0$. Choosing a much brighter magnitude limit would leave us with too few member galaxies per cluster, while choosing a much fainter magnitude limit would leave us with too few clusters sampled down to the chosen limit. Our final samples contain 63 (PBBR sample) and 261 (C4 sample) clusters; all their $\sigma_{v}$-estimates are based on $\geq 20$ galaxies.

The AGN classification of our cluster members is taken from Brinchmann et al. (hereafter B04; see also Kauffmann et al. 2003), which identify AGNs on the basis of the Baldwin et al. (1981, hereafter BPT) criteria. BPT demonstrated that it is possible to distinguish AGNs from normal star-forming galaxies by considering the intensity ratios of two pairs of relatively strong emission lines, $y \equiv[\mathrm{OIII}] \lambda 5007 / \mathrm{H} \beta$ and $x \equiv \mathrm{NII} \lambda 6583 / \mathrm{H} \alpha$. According to B04, all the galaxies with $\log y \geq 0.61 /(\log x-$ $0.47)+1.19$ (i.e. all objects above the upper line in the BPT diagram shown in Fig. 1 of B04) are classified AGNs. This classification is valid when the signal-to-noise of all the four lines involved in the diagnostic is $\geq 3$. Galaxies with a lower signalto-noise of either the [OIII] $\lambda 5007$, or the $\mathrm{H} \beta$ line (or both) are classified AGNs if $x>0.6$. AGN-classified galaxies have $>40 \%$ of their $\mathrm{H} \alpha$ luminosity contributed by an AGN (B04).

We define $f_{\mathrm{AGN}}$ as the fraction of AGNs among all the cluster members within $r_{200}$ and with $M_{r} \leq-20.0$. Since the completeness level is rather high for $M_{r} \leq-20.0$, and since the sample we use is not biased in favor or against the selection of emission-line galaxies, we deem it unnecessary to apply an incompleteness correction to the derived values of $f_{\mathrm{AGN}}$.

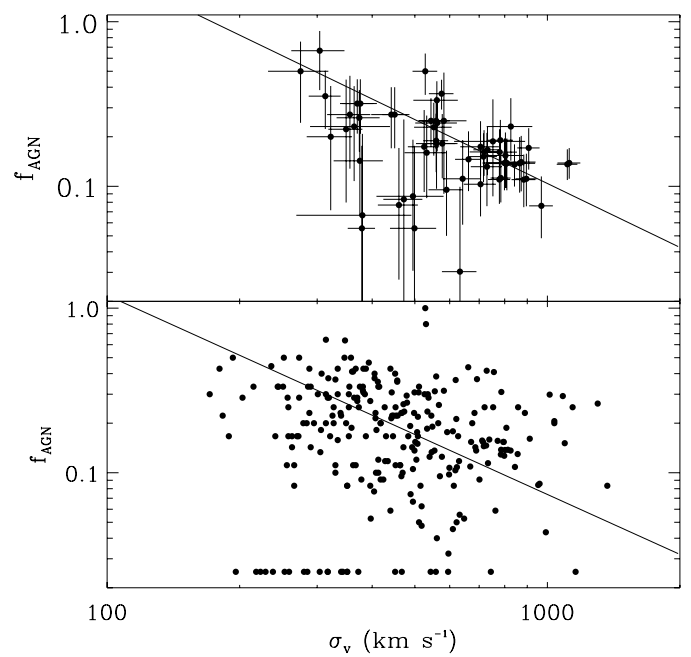

Fig. 1. Top panel: the $f_{\mathrm{AGN}}-\sigma_{v}$ diagram in logarithmic scale, for the 63 clusters of the PBBR sample. The solid line is the best-fit orthogonal linear regression in logarithmic space. 1- $\sigma$ error-bars are determined following Gerhels (1986). Bottom panel: same as panel a, for the 261 clusters of the $\mathrm{C} 4$ sample. Error-bars are not shown for the sake of clarity, but are comparable to those shown in panel a. The 23 clusters with null values of $f_{\mathrm{AGN}}$ are plotted at $f_{\mathrm{AGN}}=0.025$.

\section{Analysis and results}

The $f_{\mathrm{AGN}}$ vs. $\sigma_{v}$ diagram is shown in Fig. 1 for both cluster samples. There is a significant anti-correlation between the two quantities. The Spearman rank correlation coefficient (e.g. Press et al. 1986) is -0.45 for the PBBR sample, and the null hypothesis of no correlation is rejected with a probability of 0.9998 . The $f_{\mathrm{AGN}}-\sigma_{v}$ anti-correlation is confirmed by the analysis of the C4 sample (the Spearman correlation coefficient is -0.16 , the no-correlation hypothesis is rejected with a probability of 0.99 ). Clusters with $\sigma_{v}$ lower and, respectively, higher than $500 \mathrm{~km} \mathrm{~s}^{-1}$ have AGN fractions of $0.21 \pm 0.03$ and $0.15 \pm 0.01$, on average, in the PBBR sample (respectively $0.21 \pm 0.01$ and $0.16 \pm 0.01$, in the $\mathrm{C} 4$ sample). We note that the $f_{\mathrm{AGN}}-\sigma_{v}$ anti-correlation is not due to the $z$-dependence of the fraction of low-luminosity AGNs in the SDSS spectroscopic sample (Kauffmann et al. 2003) since there is no significant correlation between $f_{\mathrm{AGN}} \mathrm{s}$ and the average cluster redshifts in our samples. This is expected since our clusters span a narrow $z$-range.

We performed orthogonal linear regressions between $\log f_{\mathrm{AGN}}$ and $\log \sigma_{v}$, using the ODRPACK routine (Akritas \& Bershady 1996). For the C4 sample we obtain the best-fit relation $\log f_{\mathrm{AGN}}=(-1.21 \pm 0.12) \log \sigma_{v}+(2.50 \pm 0.24)$, which is consistent (within the errors) with the relation obtained for the PBBR sample. As an alternative description of the $f_{\mathrm{AGN}}-\sigma_{v}$ relation we also considered the expression $f_{\mathrm{AGN}}=C K(u)+B$, where $u=5.4 \sigma_{0} /\left(2 \sigma_{v}\right), \sigma_{0}$ is the galaxy internal velocity dispersion, and $K(u)$ is the merging rate function derived by Mamon (1992), which scales as $\sigma_{v}^{-3}$ for high, cluster-like values of $\sigma_{v}$. We take $\sigma_{0}=160 \mathrm{~km} \mathrm{~s}^{-1}$ as typical of the $M_{r} \gtrsim-20.0$ galaxies in our sample (we use the absolute magnitude vs. $\sigma_{0}$ relation of Ziegler \& Bender 1997). Such a relation, with $C=1.25$, $B=0.145$, provides an acceptable fit to the biweight-average values of $f_{\mathrm{AGN}}$ in several $\sigma_{v}$-bins both for the PBBR $\left(\chi^{2}=6.0\right.$ for 3 degrees of freedom, dof hereafter) and the C4 sample $\left(\chi^{2}=10.2\right.$ for 6 d.o.f.; see Fig. 2; see Beers et al. 1990 for the definition of the biweight-average statistics). On the other hand a constant $f_{\mathrm{AGN}}$ model is rejected (PBBR sample: $\chi^{2}=10.2$ for 4 d.o.f.; $C 4$ sample: $\chi^{2}=18.4$ for 7 d.o.f.). We discuss the physical interpretation of this relation in Sect. 4. 


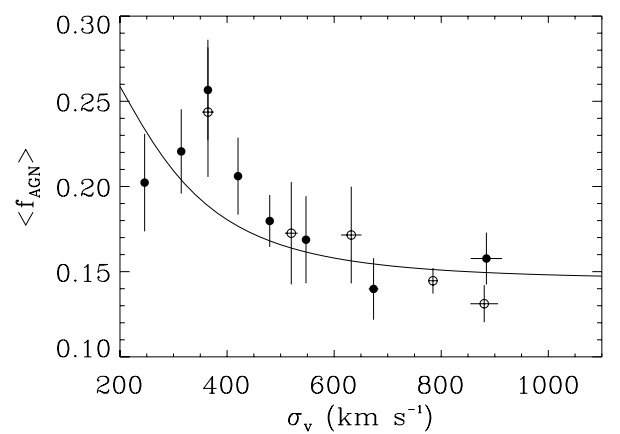

Fig. 2. Biweight-average values of $f_{\mathrm{AGN}}$ in several bins of $\sigma_{v}$ for the PBBR (open symbols) and the C4 (filled symbols) samples. The solid line displays the relation $f_{\mathrm{AGN}}=1.25 K(u)+0.145$, where $K(u)$ is the merging rate function derived by Mamon (1992), with $u=$ $5.4 \sigma_{0} /\left(2 \sigma_{v}\right)$, and $\sigma_{0}=160 \mathrm{~km} \mathrm{~s}^{-1}$.

\section{Discussion and conclusions}

We have discovered a significant anti-correlation between $f_{\mathrm{AGN}}$ and $\sigma_{v}$ in two samples of nearby galaxy clusters. Such an anticorrelation is naturally expected if the formation and/or fueling of AGNs is related to the galaxy-galaxy merging process (see, e.g., Veilleux et al. 2002; S05), since mergers of galaxies are impossible if the galaxy relative velocities are high, as in rich galaxy clusters.

The AGN fraction appears to be proportional to the merger rate in galaxy systems (as determined theoretically by Mamon 1992), plus a constant (see Fig. 2). The proportionality between $f_{\mathrm{AGN}}$ and the merger rate of cluster (or group) galaxies is expected if galaxy-galaxy mergers increase the accretion rate of the central black hole (and hence the AGN luminosity) over a time-scale similar to the merger time-scale, as shown by the simulations of S05.

Why then is $f_{\mathrm{AGN}}$ not zero for the highest- $\sigma_{v}$ clusters? Even if the selected clusters in our sample do not show significant evidence for subclustering, it is possible that most still contain undetected subclusters, characterized by rather low, group-like values of $\sigma_{v}$. Moreover, even if we have selected cluster members in projected phase-space, this does not eliminate completely the contamination by field galaxies, which Biviano et al. (2006) estimate to be $17 \%$. Hence a substantial fraction of the galaxies in our cluster samples could reside in unidentified subclusters or in the field. If $f_{\mathrm{AGN}}$ in subclusters is as high as that in compact groups (Coziol et al. 2000, 2004; Turner et al. 2001; Tovmassian et al. 2006) and if $f_{\mathrm{AGN}}$ is higher in the field than in clusters (see discussion below), this could explain why the asymptotic value of $f_{\mathrm{AGN}}$ in high- $\sigma_{v}$ clusters is not zero.

Concerning the issue of whether $f_{\mathrm{AGN}}$ in clusters is lower than $f_{\mathrm{AGN}}$ in the field, the result clearly depends on the selection of the cluster sample. We find an average $f_{\mathrm{AGN}}$ of $0.18 \pm 0.02$ for our clusters, and this is value is consistent with the lower limit obtained for the AGN fraction in the field, $f_{\text {AGN }}>0.20$ (Ho et al. 1997; Carter et al. 2001; Kauffmann et al. 2003; Miller et al. 2003; B04; Stasińska et al. 2006). On the other hand, clusters with $\sigma_{v} \geq 500 \mathrm{~km} \mathrm{~s}^{-1}$ have an average $f_{\mathrm{AGN}}$ of $0.14 \pm 0.01$, significantly lower than the field value. Our cluster AGN fraction is higher than previously reported values (Gisler 1978; Dressler et al. 1985, 1999; Hill \& Oegerle 1993; Biviano et al. 1997; Rines et al. 2005; Martini et al. 2006), but our estimate includes low-luminosity AGNs which were probably missed in the previous studies.

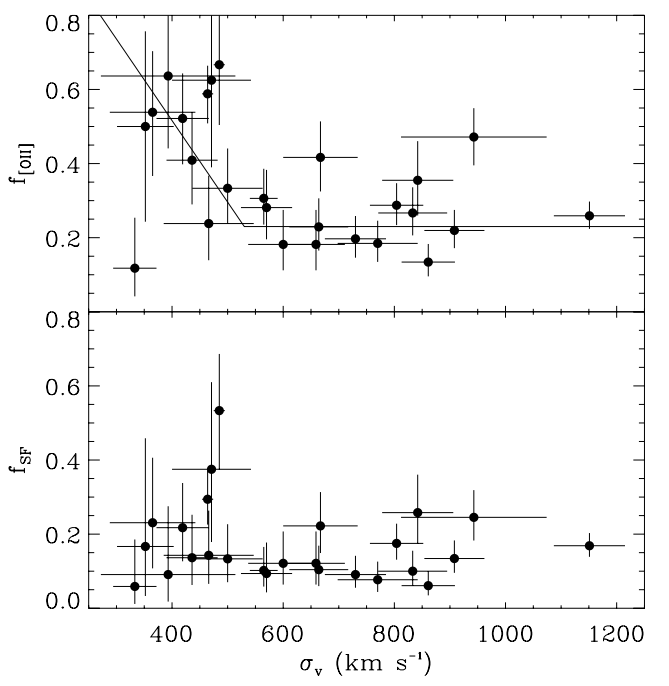

Fig. 3. The relation between $f_{[\mathrm{OII}]}\left(\right.$ top panel) and $f_{\mathrm{SF}}$ (bottom panel) and $\sigma_{v}$ for the sample of 26 clusters in common between P06's and our samples. The solid line shows the relation of P06 (see their Eq. (3)). $1-\sigma$ error bars are shown.

The $f_{\mathrm{AGN}}-\sigma_{v}$ anti-correlation is not inconsistent with the lack of a correlation between $f_{\mathrm{AGN}}$ and the galaxy number density (Miller et al. 2003). We may in fact note that the merger rate has an inverse cubic dependence on $\sigma_{v}$, but only a linear dependence on the galaxy number density. If the AGN phenomenon is indeed triggered by galaxy-galaxy mergers, the dependence of the AGN fraction on the system $\sigma_{v}$ must be easier to detect than the dependence on the density of the environment.

Our $f_{\mathrm{AGN}}-\sigma_{v}$ plot of Fig. 2 is very similar to Fig. 6 in Poggianti et al. (2006, P06 hereafter), where the fraction of cluster members with $E W(\mathrm{OII})<-3\left(f_{[\mathrm{OII}]}\right.$ hereafter $)$ is plotted as a function of the cluster $\sigma_{v}$ for a sample of 28 Abell clusters with SDSS spectroscopic data. The decreasing trend of $f_{[\mathrm{OII}]}$ with increasing $\sigma_{v}$ was interpreted by P06 in terms of a decreasing fraction of star-forming galaxies with increasing $\sigma_{v}$. Their conclusion assumes that the emission-line flux is entirely stellar in origin. While it is true that AGN and star-formation (SF hereafter) are probably related processes (e.g. S05 and references therein) it is interesting to determine whether the $f_{[\mathrm{OII}}-\sigma_{v}$ anticorrelation is driven mainly by the suppression of SF or mainly by the suppression of AGNs in high- $\sigma_{v}$ cluster galaxies.

There are 26 clusters in common between P06's sample and ours. For these clusters we plot in Fig. $3 f_{[\text {OII }]}$ vs. $\sigma_{v}$. Note that for the sake of comparison with P06, we have used only the cluster members with $E W(\mathrm{OII})<-3$. The relation of Eq. (3) in P06 is shown as a broken solid line. Clearly we reproduce their result. We then select only star-forming galaxies, following the definition of B04, i.e. excluding not only AGNs, but also all the galaxies with a composite spectrum, i.e. those for which up to $40 \%$ of their $\mathrm{H} \alpha$ luminosity might come from an AGN. The fraction of star-forming galaxies, $f_{\mathrm{SF}}$ vs. $\sigma_{v}$ is shown in the same figure. No trend is visible. The Spearman correlation coefficient is -0.05 and the null hypothesis of no correlation has an associated probability of 0.80 , hence cannot be rejected. We then conclude that the $f_{[\mathrm{OII}]}$ vs. $\sigma_{v}$ is mostly, if not entirely, due to the AGN contribution, i.e. the fraction of pure star-forming galaxies does not depend on the cluster $\sigma_{v}$ (as already found by Popesso et al. 2006b).

We conclude by pointing out that the $f_{\mathrm{AGN}}-\sigma_{v}$ relation we have found suggest that the AGN evolution must be linked to the evolution of galaxy systems. The elevated merger rate 
in low- $\sigma_{v}$ galaxy systems (groups or cluster progenitors) may produce bursts of SF in their galaxies, followed by the formation of AGNs. AGN feedback may be crucial in quenching the SF in group galaxies (Cooper et al. 2006), and in providing additional entropy to the intra-group medium (e.g. Ettori et al. 2004). The hierarchical growth of galaxy systems leads to an increase of their $\sigma_{v} \mathrm{~s}$, which suppresses the merger processes and hence the formation of AGNs (and associated feedback) in cluster galaxies.

Acknowledgements. We thank Vincenzo Mainieri and Paolo Tozzi for useful discussions. We thank the anonymous referee for suggesting us to consider the C4 cluster sample.

Funding for the SDSS and SDSS-II has been provided by the Alfred P. Sloan Foundation, the Participating Institutions, the National Science Foundation, the US Department of Energy, the National Aeronautics and Space Administration, the Japanese Monbukagakusho, the Max Planck Society, and the Higher Education Funding Council for England. The SDSS Web Site is http://www. sdss.org/. The SDSS is managed by the Astrophysical Research Consortium for the Participating Institutions. The list of Participating Institutions can be found at http://www.sdss.org/collaboration/credits.html/

\section{References}

Abazajian, K., Adelman-McCarthy, J. K., Agüeros, M. A., et al. 2004, AJ, 128, 502

Akritas, M. G., \& Bershady, M. A. 1996, ApJ, 470, 706

Baldwin, J. A., Phillips, M. M., \& Terlevich, R. 1981, PASP, 93, 5 (BPT)

Barnes, J. E., \& Hernquist, L. 1992, ARA\&A, 30, 705

Beers, T. C., Flynn, K., \& Gebhardt 1990, AJ, 100, 32

Biviano, A., Girardi, M., Giuricin, G., Mardirossian, F., \& Mezzetti, M. 1992, ApJ, 396, 35

Biviano, A., Katgert, P., Mazure, A., et al. 1997, A\&A, 321, 84

Biviano, A., Murante, G., Borgani, S., et al. 2006, A\&A, 456, 23

Brinchmann, J., Charlot, S., White, S. D. M., et al. 2004, MNRAS, 351, 115 (B04)

Cappi, M., Mazzotta, P., Elvis, M., et al. 2001, ApJ, 548, 624

Carter, B. J., Fabricant, D. G., Geller, M. J., Kurtz, M. J., \& McLean, B. 2001, ApJ, 559, 606

Cooper, M. C., Newman, J. A., Croton, D. J., et al. 2006, MNRAS, 370, 198

Coziol, R., Iovino, A., \& de Carvalho, R. R. 2000, AJ, 120, 47
Coziol, R., Brinks, E., \& Bravo-Alfaro, H. 2004, AJ, 128, 68

Dressler, A., \& Shectman, S. A. 1988, AJ, 95, 985

Dressler, A., Thompson, I. B., \& Shectman, S. A. 1985, ApJ, 288, 481

Dressler, A., Smail, I., Poggianti, B. M., et al. 1999, ApJS, 122, 51

Ettori, S., Tozzi, P., Borgani, S., \& Rosati, P. 2004, A\&A, 417, 13

Gehrels, N. 1986, ApJ, 303, 336

Girardi, M., Biviano, A., Giuricin, G., Mardirossian, F., \& Mezzetti, M. 1993, ApJ, 404, 38

Gisler, G. R. 1978, MNRAS, 183, 633

Henry, J. P., \& Briel, U. G. 1991, A\&A, 246, L14

Hill, J. M., \& Oegerle, W. R. 1993, AJ, 106, 831

Ho, L. C., Filippenko, A. V., \& Sargent, W. L. W. 1997, ApJ, 487, 568

Katgert, P., Biviano, A., \& Mazure, A. 2004, ApJ, 600, 657

Kauffmann, G., Heckman, T. M., Tremonti, C., et al. 2003, MNRAS, 346, 1055

Kauffmann, G., White, S. D. M., Heckman, T. M., et al. 2004, MNRAS, 353, 713

Lazzati, D., Campana, S., Rosati, P., Chincarini, G., \& Giacconi, R. 1998, A\&A, 331,41

Makino, J., \& Hut, P. 1997, ApJ, 481, 83

Mamon, G. A. 1992, ApJ, 401, L3

Martini, P., Kelson, D. D., Kim, E., Mulchaey, J. S., \& Athey, A. A. 2006, ApJ, 644,116

Miller, C. J., Nichol, R. C., Gómez, P. L., Hopkins, A. M., \& Bernardi, M. 2003, ApJ, 597, 142

Miller, C. J., Nichol, R. C., Reichart, D., et al. 2005, AJ, 130, 968

Poggianti, B., Von Der Linden, A., De Lucia, G., et al. 2006, ApJ, 642, 188 (P06)

Popesso, P., Biviano, A., Böhringer, H., \& Romaniello, M. 2006a, A\&A, in press [arXiv:astro-ph/0606191] (PBBR)

Popesso, P., Biviano, A., Böhringer, H., \& Romaniello, M. 2006b, A\&A, in press [arXiv: astro-ph/0609080]

Press, W. H., Flannery, B. P., Teukolsky, S. A., \& Vetterling, W. 1986, Numerical Recipes (New Yorg: Cambridge University Press)

Rines, K., Geller, M. J., Kurtz, M. J., \& Diaferio, A. 2005, AJ, 130, 1482

Springel, V., Di Matteo, T., \& Hernquist, L. 2005, ApJ, 620, L79 (S05)

Stasińska, G., Cid Fernandes, R., Mateus, A., Sodré, L. Jr., \& Asari, N. V. 2006 [arXiv:astro-ph/0606724]

Tovmassian, H., Plionis, M., \& Torres-Papaqui, J. P. 2006 [arXiv: astro-ph/0605144]

Turner, M. J. L., Reeves, J. N., Ponman, T. J., et al. 2001, A\&A, 365, L110 van Haarlem, M. P., Frenk, C. S., \& White, S. D. M. 1997, MNRAS, 287, 817

Veilleux, S., Kim, D.-C., \& Sanders, D. B. 2002, ApJS, 143, 315

York, D. G., Adelman, J., Anderson, J. E., et al. 2000, AJ, 120, 1579

Zabludoff, A. I., \& Mulchaey, J. S. 1998, ApJ, 496, 39

Ziegler, B. L., \& Bender, R. 1997, MNRAS, 291, 527 